\title{
The Peptide Network between Tetanus Toxin and Human Proteins Associated with Epilepsy
}

\author{
Guglielmo Lucchese, ${ }^{1}$ Jean Pierre Spinosa, ${ }^{2}$ and Darja Kanduc ${ }^{3}$ \\ ${ }^{1}$ Brain and Language Laboratory, Cluster of Excellence "Languages of Emotions", Free University of Berlin, 14195 Berlin, Germany \\ ${ }^{2}$ Faculty of Biology \& Medicine, University of Lausanne, CH-1011 Lausanne, Switzerland \\ ${ }^{3}$ Department of Biosciences, Biotechnologies and Biopharmaceutics, University of Bari, 70125 Bari, Italy
}

Correspondence should be addressed to Darja Kanduc; darja.kanduc@uniba.it

Received 7 March 2014; Revised 24 April 2014; Accepted 13 May 2014; Published 1 June 2014

Academic Editor: A. Vezzani

Copyright (C) 2014 Guglielmo Lucchese et al. This is an open access article distributed under the Creative Commons Attribution License, which permits unrestricted use, distribution, and reproduction in any medium, provided the original work is properly cited.

\begin{abstract}
Sequence matching analyses show that Clostridium tetani neurotoxin shares numerous pentapeptides (68, including multiple occurrences) with 42 human proteins that, when altered, have been associated with epilepsy. Such a peptide sharing is higher than expected, nonstochastic, and involves tetanus toxin-derived epitopes that have been validated as immunopositive in the human host. Of note, an unexpected high level of peptide matching is found in mitogen-activated protein kinase 10 (MK10), a protein selectively expressed in hippocampal areas. On the whole, the data indicate a potential for cross-reactivity between the neurotoxin and specific epilepsy-associated proteins and may help evaluate the potential risk for epilepsy following immune responses induced by tetanus infection. Moreover, this study may contribute to clarifying the etiopathogenesis of the different types of epilepsy.
\end{abstract}

\section{Introduction}

The term epilepsy defines a group of disturbances whose only recognized commonality is the paroxysmal synchronous discharging of groups of neurons. Localization and physiological function of the neuronal populations involved determine the clinical picture, so that (1) clinical manifestations can be extremely subtle and the diagnosis can be challenging also in terms of differential definition; (2) epilepsy(ies) can produce extremely multiform clinical pictures with a large degree of overlap [1-3]. Indeed, epileptic syndromes can also be embedded in larger syndromic clinical pictures, that is, West and Lennox-Gastaut syndromes in tuberous sclerosis complex $[4,5]$. This clinical diversity has noteworthy nosological implications. Syndromic or disease status of various forms of epilepsy and the terminology used to define them are indeed still matter of debate [7-9]. Likewise, the molecular etiopathogenesis of epilepsies has to be better defined at the molecular level. Although genetic alterations [10-12], inflammation [13], and viral infections [14-16] have been considered and thoroughly studied, nonetheless, the molecular basis and the causal mechanisms of epilepsies are still unclear.

Recently, research on epilepsy has also outlined a neurodevelopmental context [17-21]. Spontaneous recurrent seizures have been observed after induction of status epilepticus during the second and third postnatal weeks in rodents, by use of chemoconvulsants such as pilocarpine, kainate, and tetanus toxin (TT) [22]. TT seizures as well as experimental febrile seizures and developmental lithium pilocarpine appear to share a common mechanism for enhancing hippocampal network excitability and promoting epilepsy, possibly through alterations in neurotransmitter receptors or voltage-gated ion channels ([23] and further references therein).

Moreover, numerous reports suggest that immune mechanisms might play a role in processes leading to epileptogenesis [15, 24-32]. In fact, antibodies against neural antigens involved in neurotransmission have been detected in epileptic subjects [33-39], and, remarkably, epilepsy was shown to respond to immunotherapeutic approaches [38, 40, 41]. Finally, population-based cohort studies have documented 
that microbial infections during pregnancy may be a risk factor for epilepsy in offspring [42-45].

In such a multifaceted scientific-clinical context, here we analyze the peptide commonality between TT, a powerful neurotoxin used in animal models of experimental epilepsy [46-50], and human antigens that have been related to epilepsy, searching for possible immunological link(s) that might contribute to epileptogenesis. Indeed, a massive peptide overlap characterizes microbial and human proteomes [51-54] and gives grounds for questioning whether immune response(s) to microbial infections might potentially result in cross-reactions against neuronal antigens [55-58]. Pathogen versus human immune cross-reactivity might contribute to explaining the association between microbial infections and neurological syndromes [59] and assumes a special significance during pregnancy in light of the consequent possible neurodevelopmental alterations in the fetus and offspring $[26,58]$.

We report that the tetanus neurotoxin and human epilepsy antigens share an ample pentapeptide platform. The bacterial versus human peptide overlap is not random and, importantly, a search through the Immune Epitope Database (IEDB; http://www.immuneepitope.org/) reveals that the shared pentapeptides are part of TT-derived epitopes. The latter datum is relevant also in light of the role of pentapeptides as minimal functional units in cell biology and immunology $[60,61]$. On the whole, the results support the possibility that immune cross-reactions may occur between TT and epilepsy-related proteins.

\section{Methods}

TT protein sequence, UniProtKB/Swiss-Prot accession number: P04958, 1315aa long, from Clostridium tetani (NCBI Taxonomic identifier: 212717; further details at http://www .ncbi.nlm.nih.gov/Taxonomy/Browser/wwwtax.cgi) was analyzed for pentapeptide sharing with epilepsy-associated proteins as follows. First, a pentapeptide library was constructed by dissecting the TT primary sequence into pentapeptides offset by one residue, that is, MPITI, PITIN, ITINN, TINNF, INNFR, and so forth. Then, each of the final 1311 pentamers was analyzed for instances of the same match within a library consisting of primary sequences of human proteins that, when altered, have been associated with epilepsy. The number of matches and the human proteins sharing matches were recorded.

Epilepsy-associated proteins were randomly retrieved from UniProtKB Database (http://www.uniprot.org/). An unbiased set of proteins that on whatever basis (i.e., differential regulation, protein modification, or mutation) had been involved in or related to epilepsy was obtained utilizing "epilepsy" and "Homo sapiens" as keywords. Only canonical protein sequences were considered. At the time of this study, the keyword-guided search produced a library of 133 human UniProt entries, for a total of 106,022aa. Epilepsy-associated proteins are reported as UniProtKB/Swiss-Prot entry names throughout the paper, unless when discussed in detail. Any pentapeptide occurrence in the set of epilepsy-associated proteins was termed a match.
A set of proteins associated with Down syndrome, a genetic disease in which infectious agents have no role, was retrieved from UniProtKB Database and used as a comparison sample. This set was formed by the following proteins listed according to the aa length, with UniProtKB/SwissProt entries in parentheses: (1) Down syndrome critical region protein 10 (P59022, DSC10), 87aa; (2) Down syndrome critical region protein 8 (Q96T75, DSCR8), 97aa; (3) Down syndrome critical region protein 4 (P56555, DSCR4), 118aa; (4) Down syndrome critical region protein 9 (P59020, DSCR9), 149aa; (5) Down syndrome critical region protein 5 or phosphatidylinositol $\mathrm{N}$-acetylglucosaminyltransferase subunit P (P57054, PIGP), 158aa; (6) Down syndrome critical region protein 6 or protein ripply3 (P57055, DSCR6), 190aa; (7) Down syndrome candidate region 1-like 1 or regulator of calcineurin 2 (Q14206, RCAN2), 197aa; (8) Down syndrome candidate region 1-like protein 2 or regulator of calcineurin 3 (Q9UKA8, RCAN3), 24laa; (9) Down syndrome critical region protein 1 or regulator of calcineurin 1 (P53805, RCAN1), 252aa; (10) Down syndrome critical region protein 2 or proteasome assembly chaperone 1 (O95456, PSMG1), 288aa; and (11) Down syndrome critical region protein 3 (O14972, DSCR3), 297aa.

The Immune Epitope Database (IEDB; http://www.immuneepitope.org/) was used to search for TT-derived B- and/or T-cell epitopes that had been experimentally validated as positive in the human host.

Expected occurrences for pentapeptide sharing between C. tetani neurotoxin and human proteins associated with epilepsy were calculated as follows. First, we considered the number of all possible pentapeptides, $N$. Since each residue can be any of 20aa, the number of all possible pentapeptides $N$ is given by $N=20^{5}=3.2 \times 10^{5}$. Next, we considered the TT and epilepsy-associated proteins as two sets of pentapeptide size $m$ and $n$. That is, $m$ is the number of pentapeptides present in the TT protein and $n$ is the number of pentapeptides present in the epilepsy-associated protein set. If $X$ is the number of times a pentapeptide is selected in the TT protein of size $m$ and $Y$ is the number of times the same pentapeptide is selected in the epilepsy-associated protein set, then $X=m / N$ and $Y=n / N$. Assuming that $X$ and $Y$ are independent, $X Y=m n / N^{2}$. In other words, the expected number of times that one pentapeptide will be selected simultaneously in both TT and epilepsy-related protein set is given by $m n / N^{2}$. Neglecting the relative abundance of aa and assuming $m \ll N$ and $n \ll N$, we obtain a formula derived by approximation where the total number of occurrences in a second sample $n$ (the epilepsy-related protein set) of pentapeptides occurring in the first sample $m$ (TT) is given by $m n / N+m / 2$.

\section{Results and Discussion}

3.1. Description of the Pentapeptide Sharing between TT and Epilepsy-Associated Proteins. Peptide sharing between TT and human epilepsy-associated proteins was analyzed using (1) the pentapeptide module as a matching probe and (2) a library consisting of 133 epilepsy-related protein sequences retrieved from UniProt (see under Methods). 
We used pentapeptides as scanning probes in sequence similarity analyses since a grouping of five aa residues may represent a minimal unit of immune recognition in cellular and humoral responses. Indeed, scientific literature indicates that an optimal peptide length for T-cell epitopes ranges between 9 and 15 residues, with the central 5-7 aa representing the specific immune recognition contacts and the flanking residues determining the binding potential to the MHC molecules [62-66]. De facto, the HFMPT pentapeptide was reported to be a minimal antigenic determinant for MHC class I-restricted T lymphocytes [65], while the KYVKQ pentapeptide was demonstrated to be a minimal antigenic determinant for CD4(+) T-cell clones [66]; in addition, the IEDB describes numerous pentapeptide epitopes capable of binding MHC molecules (e.g., epitope IEDB IDs: 5740, 7948, 11514, 25472, and 33701) and inducing T-cell proliferation (e.g., epitope IEDB IDs: 815, 40168, 47974, 59947, 107725, 107725, and 110376) (reviewed in [61]). Likewise, humoral immune recognition/reactivity unfolds around short aa motifs ([6770]; reviewed in [71]). A representative example is a report by Zeng and colleagues [70], according to which the C-terminal pentapeptide (aa sequence: GLRPG) of luteinizing hormonereleasing hormone is a dominant $\mathrm{B}$-cell epitope able to elicit a strong anti-LHRH antibody response and to discriminate between anti-LHRH antibodies present in fertile and nonfertile mice. That is, the pentapeptide GLRPG has immunogenic and antigenic properties and also discriminates antibody specificities associated with reproductive competence.

The analyzed set of 133 human proteins related to epilepsy is listed in Box 1 according to the aa size (i.e., from IR3IP or immediate early response 3 -interacting protein 1, 82aa, to GPR98 or monogenic audiogenic seizure susceptibility protein 1 homolog, 6306aa).

Following matching analyses, we found that 42 out of the 133 epilepsy-associated proteins retrieved at random from UniProt database share 58 pentapeptides (68 including multiple occurrences) with the bacterial toxin. Box 2 lists the epilepsy-related proteins that share pentapeptides with TT and the shared pentapeptides. No TT pentapeptide match was found in the comparison set of proteins associated with Down syndrome.

\subsection{Nonstochasticity of the Pentapeptide Sharing between TT} and Epilepsy-Associated Proteins. The comparative analysis of Boxes 1 and 2 highlights three main points. Firstly, the 68 TT pentapeptide overlap described in Box 2 exceeds the expected value. As detailed under Methods, the expected number of TT pentapeptides that may occur in the epilepsyrelated protein set is given by $m n / N+m / 2$, where $m$ is the number of pentapeptides contained in TT $(1,311), n$ is the number of pentapeptides contained in the epilepsyrelated protein set $(105,490)$, and $N$ is the number of all possible pentapeptides $\left(20^{5}\right)$. Developing the equation gives 43 as expected number of pentapeptide matches, whereas the observed value is 68 (see Box 2). That is, the pentapeptide overlap between TT and epilepsy-related proteins is 1.58 times higher when compared to the expected one.

A second point of note is that the distribution of the pentapeptide overlap through the epilepsy-related proteins is unexpected. According to equation described above, pentapeptide sharing between two samples is as a quantity directly proportional to the number of pentapeptides in the analyzed samples; that is, it is proportional to the protein aa size. Actually, 91 epilepsy-related proteins are excluded from the pentapeptide matching with TT, independently of their length. For example, SPTN1, 2472aa (see Box 1), has no bacterial matches, while LRRC1, 524aa, shares 3 pentapeptides with TT (Box 2).

In summary, a comparative analysis of Boxes 1 and 2 highlights that 68 TT pentapeptide matches are allocated in 42 out 133 human proteins that have been related, when altered, to epilepsy, and no relationship appears to exist between pentapeptide sharing and the human protein size. Applying the equation described above to the set of 42 epilepsy-related proteins sharing 68 pentapeptides with TT and amounting to $50,254 \mathrm{aa}$, the expected pentapeptide overlap is equal to 20 , so that the observed occurrence value is 3,4 times higher.

Finally, a third punctum saliens is that nonrandomness characterizes also the distribution of the TT pentapeptides among the 42 epilepsy-associated proteins. Box 2 shows that a few TT pentapeptides are repeated in the 42 epilepsy-associated protein set. Indeed, TT pentapeptides EIIPS, SLSIG, and FCKAL recur twice, and TT pentapeptides FGGQD, KEIEK, and TFLRD occur three times (Box 2; see pentapeptides underlined). Box 2 also shows that MK10 (mitogen-activated protein kinase 10; 464aa); CDKL5 (cyclin-dependent kinase-like 5; 1030aa); and KCMA1 (calcium-activated potassium channel subunit alpha-1; 1236aa) share two sequentially overlapping pentapeptides with TT, that is, share the hexapeptides SVDDAL, KNSFSE, and PKEIEK, respectively. The nonrandom TT pentapeptide sharing clearly emerges from Figure 1, where expected and observed occurrence values are graphically compared.

It can be seen that, in conflict with the theoretical trend of the TT pentapeptide matching as a function of epilepsyrelated protein length (Figure 1, columns in gray), the observed to expected ratio of pentapeptide matching shows no relationship with the human protein length (Figure 1, columns in black). For example, contrary to mathematical expectations, MK10 (464aa long) has three pentapeptide matches, whereas VP13A (3174aa long) has one match (see Box 2 and Figure 1).

\subsection{Immunologic Potential of the Pentapeptide Sharing} between TT and Epilepsy-Associated Proteins. Having defined the TT versus epilepsy-associated proteins pentapeptide overlap, it was next tested whether such a sharing has an immunologic potential. To this aim we used IEDB, a database that describes B- and T-cell epitopes for humans, nonhuman primates, rodents, and other animal species, and searched for TT-derived epitopes that had been validated as immunopositive in humans. At the time of the search, we obtained a list of 517 TT-derived epitopes. The pentapeptides common to epilepsy-associated proteins and TT (see Box 2, sequences in italic) were used as probes to scan the 517 TTderived epitope set in order to define potential cross-reactive peptide sequences. Results are reported in Table 1. 
IR3IP (82); CYTB (98); MPC1 (109); OPALI (141); ARF6 (175); DYR (187); CP013 (204); GOSR2 (212); RB39B (213); SCN1B (218); THEM4 (240); FOLR1 (257); PNPO (261); CLN8 (286); ROGDI (287); KCTD7 (289); SEN34 (310); CLN6 (311); PPR3C (317); PRS41 (318); GHC1 (323); EPM2A (331); PRRT2 (340); EP2A2 (344); RENR (350); MPRB (354); CLN5 (358); PHF6 (365); LIAS (372); IRK10 (379); CYB (380); AMACR (382); IRK11 (390); NHLC1 (395); ASAH1 (395); SNIP1 (396); ARC (396); NAGAB (411); BCKD (412); SIAT9 (418); STRAA (431); CBPA6 (437); DCX (441); PH4H (452); IDHP (452); GBRD (452); GBRA1 (456); NDUV1 (464); MK10 (464); SRPX2 (465); SEN2 (465); GBRG2 (467); MEF2C (473); GBRB3 (473); SCRB2 (478); AKT1 (480); PUR8 (484); GTR1 (492); KCNA1 (495); KCNV1 (500); ACHB2 (502); ARHG9 (516); JERKY (520); CACB4 (520); D2HDH (521); LRRC1 (524); SEN54 (526); ACHA2 (529); LGI4 (537); AL7A1 (539); LGI2 (545); LGI3 (548); LGI1 (557); TBC24 (559); ARX (562); TPP1 (563); GGT3 (568); PUR9 (592); STXB1 (594); DCE1 (594); EPMIP (607); LBR (615); ACHA4 (627); EFHC1 (640); SL9A9 (645); ITF2 (667); TSEAR (669); SL9A6 (669); SYN1 (705); EFHC2 (749); TRM44 (757); AFG32 (797); BRAT1 (821); PRIC1 (831); PRIC2 (844); KCNQ3 (872); KCNQ2 (872); SOBP (873); MANBA (879); CLCN2 (898); PWP2 (919); GABR1 (961); MIB1 (1006); CDKL5 (1030); AT2A2 (1042); DLGP2 (1054); CASR (1078); AP4E1 (1137); PCD19 (1148); TSC1 (1164); ATN1 (1190); ZEB2 (1214); PLCB1 (1216); KCMA1 (1236); DPOG1 (1239); TPC10 (1259); CNTP2 (1331); ARHGA (1369); NMDE1 (1464); WDR62 (1518); ABCC8 (1581); GCP6 (1819); SCN8A (1980); SCN9A (1988); SCN2A (2005); SCN1A (2009); CAC1E (2313); CAC1H (2353); SPTN1 (2472); VP13A (3174); RELN (3460); CSMD3 (3707); GPR98 (6306)

Box 1: List of the 133 epilepsy-associated proteins analyzed for TT pentapeptide sharing. Proteins were randomly retrieved from UniProtKB (http://www.uniprot.org/) as described under Methods. Proteins are indicated by UniProtKB/Swiss-Prot entry names, and listed according to increasing aa length reported in parentheses.

ROGDI (LKDKI); CLN6 (PALLL); CLN5 (VIVHK); NHLC1 (TITND); ASAH1 (PVLNK); CBPA6 (GIPYA);

GBRA1 (VSFWL); MK10 (SVDDAL*, GAQGI); GTR1 (SYLSI); ARHG9 (KLEEK); D2HDH (GSGLV);

LRRC1 (NKNEV, SLTDL, FCKAL); ACHA2 (NITSL, EIIPS); LGI2 (KAKWL); LGI1 (GFTEI); EPMIP (SGLVS);

ACHA4 (EIIPS); EFHC1 (RVPKV); SL9A9 (IMYGF); TSEAR (QGYEG); SL9A6 (SIMYG, SPTTL); EFHC2

(GDFIK); AFG32 (DPALL); CLCN2 (TFRDL); PWP2 (GQYIV, HSLSI); GABR1 (EDIDV); CDKL5

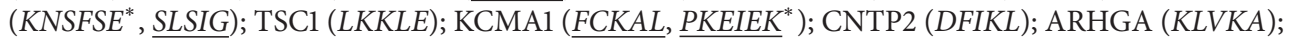

NMDE1 (PALNI); WDR62 (LIHVL, KRSYQ, TNGKL); GCP6 (SLSIG, KEIEK); SCN8A (FGGQD, DTQSK,

LRVPK, TFLRD); SCN9A (NDMFN); SCN2A (FGGQD, TFLRD); SCN1A (FGGQD, TFLRD); VP13A

(ITMTN); RELN (SIGSG); CSMD3 (EGFNI, KEIEK); GPR98 (LISID, ESKDL, VDGSG, TLPND, SGFNS, LSSAN, VQLKN)

Box 2: Peptide sharing between TT and epilepsy-associated proteins. Proteins reported by UniProtKB/Swiss-Prot entry names and listed according to the aa length. Pentapeptides shared with TT are italic in parentheses. Pentapeptides present more than once in the epilepsy antigen set are underlined. Sharing of two consecutively overlapped pentapeptides (i.e., a hexapeptide) is indicated by an asterisk.

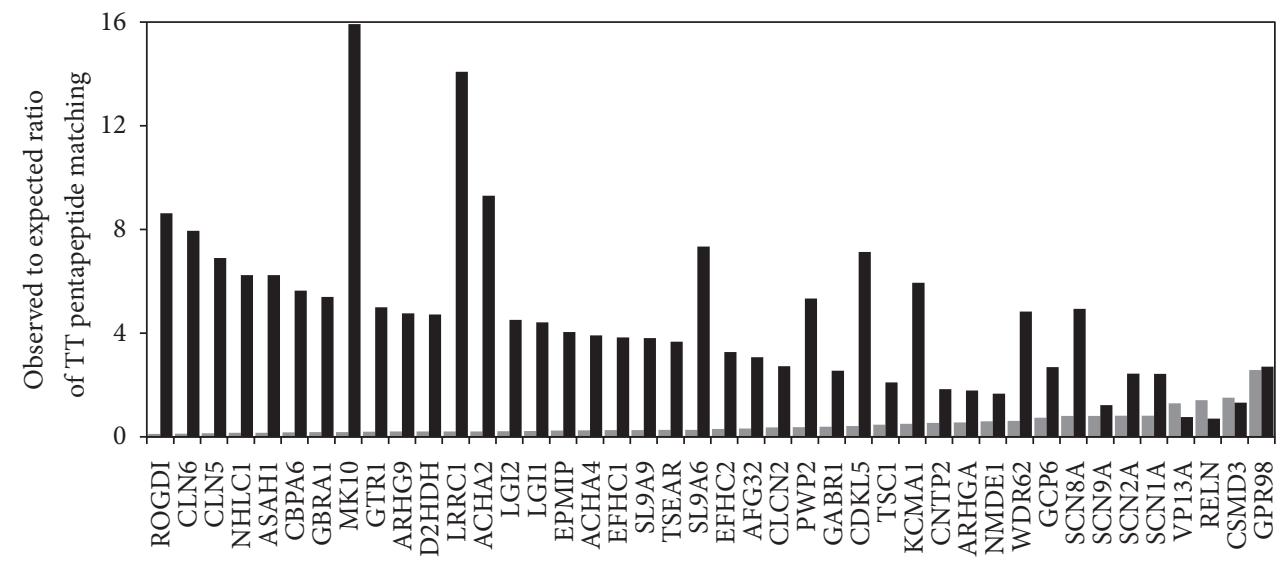

Epilepsy-associated proteins

FIGURE 1: Observed versus expected pentapeptide matching between TT and epilepsy-related proteins. The 42 proteins sharing pentapeptides with TT are allocated along the $x$-axis according to increasing aa length. Gray columns: expected matches calculated according to the formula $m n / N+m / 2$, where $m$ is the number of pentapeptides present in the neurotoxin $(1,311)$ and $n$ is the number of pentapeptides present in the epilepsy-associated protein (see Methods). For example, in the case of IR3IP protein, 82aa, the possible pentapeptide overlap is equal to 1,311 $\times 78 / 3,200,000+1,311 / 2$. Black columns: observed to expected ratio of the pentapeptide matching. Observed matching values from Box 2 . 
In essence, Table 1 shows that all of the 58 pentapeptides common to the 42 epilepsy-associated proteins and TT (Box 2, peptide sequences in parentheses and in italic) are present in 116 TT-derived epitopes that had been established to be immunopositive in humans. This datum indicates a potential vulnerability of the 42 epilepsy-associated proteins to cross-reactions following anti-TT immune responses. Moreover, many TT-derived epitopes share fragments with distinct epilepsy-related proteins and are of particular significance to a multiple cross-reactivity risk, since, for example, an immune response targeting the TT epitope fnnftVSFWLRVPKVsahle (see Table 1, IEDB ID 17207, with shared fragments in capital letter) has the potential to cross-react with the following three crucial proteins related to different forms of epilepsy:

(i) GBRA1 or gamma-aminobutyric acid receptor subunit alpha-1, the major inhibitory neurotransmitter in the vertebrate brain that mediates neuronal inhibition by binding to the GABA/benzodiazepine receptor and opening an integral chloride channel [72],

(ii) SCN8A or voltage-gated sodium channel subunit alpha Navl.6, a protein that mediates the voltagedependent sodium ion permeability of excitable membranes [73],

(iii) EFHC1 or myoclonin-1, a protein that may enhance calcium influx through CACNA1E and stimulate programmed cell death [74].

Such a multiple cross-reactivity potential is shown also by other TT-derived epitopes, eg, epitopes IEDB IDs 30436, 48049,113407 , and so forth.

Also, it seems important to highlight that MK10 (mitogen-activated protein kinase 10, also known as stressactivated protein kinase JNK3 or p493F12 kinase), a protein that shows the highest unexpected level of pentapeptide overlap to TT (Figure 1) and also has a high immunologic potential as illustrated in Table 1 (i.e., MK10 pentapeptide(s) are present in 7 TT-derived epitopes), is selectively expressed in a subpopulation of pyramidal neurons in the CA1, CA4, and subiculum regions of the hippocampus, and layers 3 and 5 of the neocortex [75]. That is, there is a potential cross-reactivity risk specifically allocated in brain areas directly linked to epileptogenesis $[76,77]$.

\section{Conclusions}

This study describes a vast pentapeptide commonality between TT-derived epitopes and epilepsy-associated proteins. This peptide sharing acquires a relevant pathologic potential in light of the fact that pentapeptide modules have the capacity of inducing immune response(s) and are main players in immune recognition [61-71]. Immunologically, two sequences that share a pentapeptide are potentially subject to a cross-reaction [60].

In the disease model examined here, that is, tetanus infection and epilepsy, the ample cross-reactivity platform between TT-derived epitopes and human epilepsy-associated antigens supports the hypothesis of an immune involvement in epilepsy. As a matter of fact, all the 42 epilepsy-related proteins listed in Box 2 are potential targets of cross-reactions (see Table 1). Qualitatively, the peptide overlap occurs in human proteins canonically associated with epilepsy such as gamma-aminobutyric acid receptor subunit alpha1 (GBRA1), gamma-aminobutyric acid type B receptor subunit 1 (GABR1), sodium channel protein subunits (SCN1A, SCN2A. SCN8A, and SCN9A), and calcium-activated potassium channel subunit alpha-1 (KCMA1) (Table 1). Obviously, an immune attack against such epilepsy-associated proteins may cause alterations to neural structures and functions, especially when the neurodevelopmental intrauterine phase is considered. Being of nonsecondary importance, the nonstochastic character of the peptide overlap between TT and epilepsy-associated proteins (Figure 1) indicates that the potential cross-reactivity extent (and the associated risk of developing epilepsy and neurodevelopmental disorders) will increase with the number of anti-TT immune stimulations.

An additional relevant point is the "antigenic patchwork" shown in Table 1. Indeed, the potential peptide crossreactome involved in different extent and in different combinations of 42 epilepsy-associated proteins might help understand the complex neurobiological network that, once hit and perturbed, may underlie different epileptic forms [1-9]. Also, it has to be noted that Table 1 includes proteins such as CNTP2 or contactin-associated protein-like 2, RELN or reelin, and TSC1 or tuberous sclerosis 1 protein, which are also landmark antigens for autism and the associated impairment in communication/language skills and behaviors [78-81]. Hence, Table 1 may provide a mechanistic framework to allocate the occurrence of epilepsy, intellectual disability, and autism spectrum disorder in patients with tuberous sclerosis complex. Likewise, data from Table 1 might contribute to answering a critical question in neuropsychopathology, that is, the coexistence of patients with combined schizophrenia and epilepsy [82-85]. Indeed, Table 1 substantiates the hypothesis according to which the thread joining epilepsy and schizophrenia may reside in neurodevelopmental molecules such as leucine-rich glioma inactivated (LGI) proteins and GPR98, a G protein-coupled receptor, originally known as VLGR1 or very large G protein-coupled receptor [86]. De facto, Table 1 shows that fragments from LGI1, LGI2, and GPR98 are present in 1, 7, and 18 TT-derived epitopes, respectively. In other words, the potential cross-reactivity targeting LGI1, LGI2, and GPR98 following an anti-TT response is high.

Given the caveat that peptide immunoreactivity is influenced by numerous factors, for example, binding affinity [87], cripticity (i.e., determinants embedded in membrane structures do not induce immune responses under physiological conditions) [88], and posttranslational modifications (i.e., citrullination) [89], the present data might contribute to further our understanding of epilepsies. In particular, data from Table 1 might represent a peptide platform to be tested in antibody binding assays using sera from epileptic subjects. Accompanied by parallel immunoassays based on the utilization of epilepsy-related proteins as antigens, such an approach might not only validate the TT-epilepsy link proposed in this study, but also lead to a definition at 
TABLE 1: Pentapeptide sharing between TT-derived epitopes and human epilepsy-associated proteins.

\begin{tabular}{|c|c|c|c|}
\hline IEDB ID $^{1}$ & TT-derived epitope $\mathrm{e}^{2,3}$ & Immune context & Epilepsy-associated proteins ${ }^{4}$ \\
\hline 1270 & afcpeyvptfdnvieNITSL & HLA-Class II, allele undetermined & ACHA2 \\
\hline 1389 & afrnVDGSGLVSklig & HLA-Class II, allele undetermined & GPR98 D2HDH EPMIP \\
\hline 1501 & agevrqiTFRDLpdkfnayl & HLA-Class II, allele undetermined & CLCN2 \\
\hline 1929 & aihlvnnesseVIVHKamdi & HLA-DRB1 ${ }^{*}$ 04:01 & CLN5 \\
\hline 2219 & akkqllefDTQSKnilmqyi & HLA-Class II, allele undetermined & SCN8A \\
\hline 3156 & amltnliifgpgPVLNKNEV & HLA-Class II, allele undetermined & ASAH1 LRRC1 \\
\hline 3418 & anskfigiteLKKLEskink & HLA-DRB1*11:01 & TSC1 \\
\hline 3832 & apsyTNGKLniyyrrlyngl & HLA-DRB5 $^{*}$ 01:01, HLA-DRB1 ${ }^{*} 13: 01$ & WDR62 \\
\hline 7603 & danLISIDikndlyektl & HLA-DRB1 ${ }^{*}$ 03:01 & GPR98 \\
\hline 8734 & dinndiisdiSGFNSsvity & HLA-DRB1 ${ }^{*} 01: 01$ & GPR98 \\
\hline 8778 & diSGFNSsvitypdaqlvpg & HLA-DRB1*15:01 & GPR98 \\
\hline /8903 & dkisdvstivpyigPALNIv & HLA-DPB1* 04:01, HLA-DRB1 ${ }^{*} 15: 01$ & NMDE1 \\
\hline 9297 & dltfiaeKNSFSEepfqdei & HLA-DRB1* 01:01, HLA-DRB1* 04:01 & CDKL5 \\
\hline 9595 & DPALLLmheLIHVLhglyg & $\begin{array}{l}\text { B-cell } \\
\text { HLA-DR2; HLA-Class II, allele } \\
\text { undetermined }\end{array}$ & AFG32 CLN6 WDR62 \\
\hline 9595 & drLSSANlyingvlmgsaei & $\begin{array}{l}\text { B-cell } \\
\text { HLA-DR2; HLA-Class II, allele } \\
\text { undetermined }\end{array}$ & GPR98 \\
\hline 10472 & DTQSKnilqyikanskfigiteLKKLEski & HLA-Class II, allele undetermined & SCN8A TSC1 \\
\hline 11980 & efDTQSKnilmqyikanskfigitel & B-cell & SCN8A \\
\hline 13095 & eLIHVLhglygmqvss & $\begin{array}{l}\text { B-cell } \\
\text { HLA-DR2; HLA-Class I, allele } \\
\text { undetermined }\end{array}$ & WDR62 \\
\hline 13125 & eLKKLEskinkvfstpipfs & HLA-Class II, allele undetermined & TSC1 \\
\hline 13813 & eqdpsgattksamltnliifgpgPVLNKNEV & HLA-Class II, allele undetermined & ASAH1 LRRC1 \\
\hline 15087 & eysiessmkkHSLSIGSGwsvsl & B-cell & PWP2 GCP6 CDKL5 RELN \\
\hline 15411 & fdkdsnGQYIVnedkfqily & HLA-Class II, allele undetermined & PWP2 \\
\hline 16155 & fiaeKNSFSEepfqdeivsyntk & B-cell & CDKL5 \\
\hline 17134 & fnaylankwvfiTITNDrls & HLA-Class II, allele undetermined & NHLC1 \\
\hline 17205 & fnnftVSFWLRVPK & HLA-Class II, allele undetermined & GBRA1 SCN8A \\
\hline 17206 & fnnftVSFWLRVPKVsahle & HLA-DR3 & GBRA1 SCN8A EFHC1 \\
\hline 17207 & fnnftVSFWLRVPKVsashle & $\begin{array}{l}\text { HLA-DRB1*11:01, HLA-DR, HLA-DR1, } \\
\text { HLA-DR5, HLA-DR7, HLA-DR11, } \\
\text { HLA-DPw4, HLA-Class II, allele } \\
\text { undetermined }\end{array}$ & GBRA1 SCN8A EFHC1 \\
\hline 17208 & fnnftVSFWLRVPKVsashleqy & $\begin{array}{l}\text { HLA-DRB1 }^{*} \text { 01:01, HLA-DRB1 } \\
\text { HLA-HLA-DRB1 } \\
\text { HL 07:01, HLA-DRB1 }\end{array}$ & GBRA1 SCN8A EFHC1 \\
\hline 17487 & fqilynSIMYGFTEIelgkk & HLA-Class II, allele undetermined & SL9A6 SL9A9 LGI1 \\
\hline 18217 & fvksGDFIKLyvsynnnehivgy & B-cell & EFHC2 CNTP2 \\
\hline 18356 & fwLRVPKVsashleqygtne & HLA-DRB1* 11:01 & SCN8A EFHC1 \\
\hline 19469 & gevrqiTFRDLpdkfnaylankw & B-cell & CLCN2 \\
\hline 21599 & gpdkeqiadeinnlknKLEEKan & B-cell & ARHG9 \\
\hline 22769 & gtneysiissmkkHSLSIGS & DQB1 $^{*} 06: 02, \mathrm{DRB}^{*} 01: 01$ & PWP2 GCP6 CDKL5 \\
\hline 24238 & hLKDKIlgcdwyfvptdegwtnd & HLA-Class II, allele undetermined & ROGDI \\
\hline 25597 & idkisdvstivpyigPALNI & HLA-Class II, allele undetermined & NMDE1 \\
\hline 25666 & idsfvksGDFIKLyvsynnn & HLA-DRB1 ${ }^{*} 15: 01$ & EFHC2 CNTP2 \\
\hline 26808 & ikiknedltfiaeKNSFSEe & HLA-Class II, allele undetermined & CDKL5 \\
\hline 27639 & ingkaihlvnnesseVIVHK & HLA-Class II, allele undetermined & CLN5 \\
\hline
\end{tabular}


TABle 1: Continued.

\begin{tabular}{|c|c|c|c|}
\hline IEDB ID $^{1}$ & TT-derived epitope $e^{2,3}$ & Immune context & Epilepsy-associated proteins ${ }^{4}$ \\
\hline 29241 & ivdynlqskiTLPNDrttpv & HLA-Class II, allele undetermined & GPR98 \\
\hline 29331 & ivkQGYEGnfig & HLA-Class II, allele undetermined & TSEAR \\
\hline 29407 & ivpyigPALNIv & HLA-Class II, allele undetermined & NMDE1 \\
\hline 29408 & ivpyigPALNIvkQGYEGnf & HLA-DRB1 ${ }^{*} 15: 01$ & NMDE1 TSEAR \\
\hline 29843 & KAKWLgtvntqfqKRSYQ & HLA-Class II, allele undetermined & LGI2 WDR62 \\
\hline 29891 & kamdieyNDMFNnftVSFWLrvp & B-cell & SCN9A GBRA1 \\
\hline 30269 & kdVQLKNitdymyltnapsy & HLA-DRB1* 01:01, HLA-DRB1* 04:01 & GPR98 \\
\hline 30436 & KEIEKlytSYLSITFLRDpwgnp & B-cell & $\begin{array}{l}\text { CSMD3 KCMA1 GCP6 GTR1 } \\
\text { SCN1A SCN2A SCN8A CLCN2 }\end{array}$ \\
\hline 30572 & keqiadeinnlknKLEEKan & HLA-Class II, allele undetermined & ARHG9 \\
\hline 32521 & knitdymyltnapsyTNGKL & HLA-Class II, allele undetermined & WDR62 \\
\hline 32546 & knldcwvdneEDIDVilkkstil & B-cell & GABR1 \\
\hline 33527 & kstilnldinndiisdiSGFNSs & B-cell & GPR98 \\
\hline 34301 & kwievyKLVKAKWLgtvntq & HLA-DRB1 ${ }^{*} 01: 01$ & ARHGA LGI2 \\
\hline 34887 & lankwvfiTITNDrLSSANlyin & B-cell & NHLC1 GPR98 \\
\hline 35058 & lcikiknedltfiaeKNSFS & HLA-DRB1 ${ }^{*}$ 04:01 & CDKL5 \\
\hline 35566 & lekryekwievyKLVKAKWL & HLA-Class II, allele undetermined & ARHGA LGI2 \\
\hline 35993 & lftFGGQDanLISIDikndl & HLA-Class II, allele undetermined & SCN1A SCN2A SCN8A GPR98 \\
\hline 36667 & lipvassskdVQLKNitdym & HLA-DRB1*11:01 & GPR98 \\
\hline 38977 & lqrITMTNSVDDALinstki & HLA-Class II, allele undetermined & VP13A MK10 \\
\hline 40770 & lygmqvsshEIIPSkqeiym & HLA-Class II, allele undetermined & ACHA2 ACHA4 \\
\hline 41527 & mfnnftVSFWLRVPKVsash & HLA-DRB1*11:01 & GBRA1 SCN8A EFHC1 \\
\hline 42847 & mtnSVDDALinstkiysyfp & HLA-DRB1*11:01 & MK10 \\
\hline 43280 & napsyTNGKLniyyrrlynglkf & B-cell & WDR62 \\
\hline 43519 & ndrLSSANlyingvlmgsae & HLA-Class II, allele undetermined & GPR98 \\
\hline 43591 & neEDIDVilkkstilnldin & HLA-Class II, allele undetermined & GABR1 \\
\hline 43939 & nftVSFWLRVPK & HLA-Class II, allele undetermined & GBRA1 SCN8A \\
\hline 43940 & nftVSFWLRVPKVsashle & HLA-DRB1*11:01 & GBRA1 SCN8A EFHC1 \\
\hline 44007 & ngkaihlvnnesseVIVHKamdi & B-cell & CLN5 \\
\hline 44396 & nivkQGYEGnfi & HLA-Class II, allele undetermined & TSEAR \\
\hline 44200 & niddntiyqylyaqkSPTTL & HLA-DRB1* 01:01 & SL9A6 \\
\hline 44383 & NITSLtigkskyfqDPALLL & HLA-ClassII, allele undetermined & ACHA2 AFG32 CLN6 \\
\hline 44557 & NKNEVrgivlrvdnknyfpc & HLA-Class II, allele undetermined & LRRC1 \\
\hline 44667 & nldinndiisdiSGFNSsvi & HLA-Class II, allele undetermined & GPR98 \\
\hline 45102 & nnftVSFWLRVPKVsashle & HLA-Class II, allele undetermined & GBRA1 SCN8A EFHC1 \\
\hline 46136 & ntiyqylyaqkSPTTLqrit & HLA-Class II, allele undetermined & SL9A6 \\
\hline 46853 & PALLLmheLIHVLhglygmq & HLA-Class II, allele undetermined & CLN6 WDR62 \\
\hline 46855 & PALNIvkQGYEGnfigalet & HLA-Class II, allele undetermined & NMDE1 TSEAR \\
\hline 48049 & PKEIEKlytSYLSITFLRDf & HLA-Class II, allele undetermined & $\begin{array}{l}\text { GCP6 CSMD3 KCMA1 GTR1 } \\
\text { SCN1A SCN2A SCN8A CLCN2 }\end{array}$ \\
\hline 48697 & pnrdiliasnwyfnhLKDKIlgc & B-cell & ROGDI \\
\hline 49984 & pvtkGIPYApeyksnaastteih & B-cell & CBPA6 \\
\hline 51254 & qkSPTTLqrITMTNSVDDALIns & B-cell & SL9A6 VP13A MK10 \\
\hline 56528 & ryekwievyKLVKAKWLgtvntq & B-cell & ARHGA LGI2 \\
\hline 57935 & sfvksGDFIKLyvsynnneh & HLA-ClassII, allele undetermined & EFHC2 CNTP2 \\
\hline 57947 & sfwLRVPKVsashle & HLA-DR5, HLA-DRB1*11:01 & SCN8A EFHC1 \\
\hline 58527 & SIGSGwsvslkgnnliwtlk & HLA-DRB1 ${ }^{*}$ 03:01 & RELN \\
\hline 59500 & SLTDLggelcikikn & HLA-Class II, allele undetermined & LRRC1 \\
\hline
\end{tabular}


TABle 1: Continued.

\begin{tabular}{|c|c|c|c|}
\hline IEDB ID $^{1}$ & TT-derived epitope $e^{2,3}$ & Immune context & Epilepsy-associated proteins ${ }^{4}$ \\
\hline 61214 & ssmkkHSLSIGSGwsvslkg & HLA-Class II, allele undetermined & PWP2 GCP6 CDKL5 RELN \\
\hline 61354 & ssskdVQLKNitdymyltnapsy & B-cell & GPR98 \\
\hline 62073 & SVDDALinstkiysyfpsviskvnqGAQGIl & HLA-Class II, allele undetermined & MK10 \\
\hline 63277 & tdymyltnapsyTNGKLniy & HLA-DRB1* 01:01, HLA-DRB1* 04:01 & WDR62 \\
\hline 63450 & teLKKLEskinkvfstpipf & HLA-DRB1* 07:01 & TSC1 \\
\hline 64514 & tiyndtEGFNIESKDLksey & HLA-Class II, allele undetermined & CSMD3 GPR98 \\
\hline 65324 & TNGKLniyyrrlynglkfii & HLA-Class II, allele undetermined & WDR62 \\
\hline 67104 & tvntqfqKRSYQmyrsletqvda & B-cell & WDR62 \\
\hline 67147 & tVSFWLRVPKVsa & HLA-DRB1*11:01, HLA-DRB1*11:04 & GBRA1 SCN8A EFHC1 \\
\hline 67148 & tVSFWLRVPKVsashle & HLA-DRB1* 11:01 & GBRA1 SCN8A EFHC1 \\
\hline 68104 & vdynlqskiTLPNDrttpvt & HLA-DQB1 ${ }^{*}$ 06:02 & GPR98 \\
\hline 69149 & VIVHKamdieyNDMFNnftv & HLA-Class II, allele undetermined & CLN5 SCN9A \\
\hline 69180 & vKAKWLgtvntqfqKRSYQm & HLA-DQB1*06:02 & LGI2 WDR62 \\
\hline 70202 & vntqfqKRSYQmyrsleyqv & HLA-DRB1* 07:01 & WDR62 \\
\hline 70165 & vnqGAQGIlflqwvrdiidd & HLA-Class II, allele undetermined & MK10 \\
\hline 70166 & vnqGAQGIlflqwvrdiiddftn & B-cell & MK10 \\
\hline 70514 & vpyigPALNIvk & HLA-Class II, allele undetermined & NMDE1 \\
\hline 70982 & vsidkfriFCKALnpk & HLA-DRB1* 11:01 & LRRC1 SCN8A KCMA1 \\
\hline 71155 & vstivpyigPALNI & HLA-DR, HLA-DR1, HLA-A*02:01 & NMDE1 \\
\hline 71156 & vstivpyigPALNIvkQGYEGnf & B-cell & NMDE1 TSEAR \\
\hline 72784 & wLRVPKVsashleqygtneysie & B-cell & SCN8A EFHC1 \\
\hline 76411 & yvsidkfriFCKALnPKEIE & HLA-Class II, allele undetermined & $\mathrm{LRRC1}_{\mathrm{KCMA1}}{ }^{5}$ \\
\hline 76537 & yylipvassskdVQLKNitd & HLA-Class II, allele undetermined & GPR98 \\
\hline 79808 & eLIHVLhglygmq & HLA-DRA* 01:01, HLA-DRB1 ${ }^{*}$ 01:01 & WDR62 KCMA1 \\
\hline 79816 & evyKLVKAKWLgt & HLA-DRA* 01:01, HLA-DRB1 ${ }^{*}$ 01:01 & ARHGA LGI2 \\
\hline 113407 & fnnftVSFWLRVPKVsas & HLA-DR11 & GBRA1 SCN8A EFHC1 \\
\hline 167585 & glygmqvsshEIIPSkqeiy & HLA-DRB1* 12:01 & ACHA2 ACHA4 \\
\hline 167613 & kvnqGAQGIlflqwvrdiid & HLA-DRB1 ${ }^{*} 12: 01$ & MK10 \\
\hline 167626 & nLISIDikndlyektlndyk & HLA-DRB1*12:01 & GPR98 \\
\hline 167666 & shEIIPSkqeiymqhtypis & HLA-DRB1* 12:01 & ACHA2 ACHA4 \\
\hline
\end{tabular}

the molecular level of the repeatedly advanced association between antibodies and epilepsy [33-41]. Moreover, of not less importance, immunoassay validation could also represent a prelude to specific therapies based on peptide modules able to block epileptogenic anti-TT autoantibodies [38$41,90]$. Immunological research in this direction has been programmed in our lab.

\section{Conflict of Interests}

The authors declare that there is no conflict of interests regarding the publication of this paper.

\section{Acknowledgment}

Guglielmo Lucchese is supported by Deutscher Akademischer Austauschdienst (DAAD).

\section{References}

[1] C. D. Ferrie, "Idiopathic generalized epilepsies imitating focal epilepsies," Epilepsia, vol. 46, no. 9, pp. 91-95, 2005.

[2] E. C. Wirrell, B. R. Grossardt, E. L. So, and K. C. Nickels, "A population-based study of long-term outcomes of cryptogenic focal epilepsy in childhood: cryptogenic epilepsy is NOT probably symptomatic epilepsy," Epilepsia, vol. 52, no. 4, pp. 738-745, 2011. 
[3] A. T. Berg, "Epilepsy, cognition, and behavior: the clinical picture," Epilepsia, vol. 52, supplement 1, pp. 7-12, 2011.

[4] U. Stephani, "The natural history of myoclonic astatic epilepsy (Doose syndrome) and Lennox-Gastaut syndrome," Epilepsia, vol. 47, supplement 2, pp. 53-55, 2006.

[5] C. J. Chu-Shore, P. Major, S. Camposano, D. Muzykewicz, and E. A. Thiele, "The natural history of epilepsy in tuberous sclerosis complex," Epilepsia, vol. 51, no. 7, pp. 1236-1241, 2010.

[6] N. Gaspard and L. J. Hirsch, "Pitfalls in ictal EEG interpretation: critical care and intracranial recordings," Neurology, vol. 80, supplement 1, pp. S26-S42, 2013.

[7] A. T. Berg, S. F. Berkovic, M. J. Brodie et al., "Revised terminology and concepts for organization of seizures and epilepsies: report of the ILAE Commission on Classification and Terminology, 2005-2009," Epilepsia, vol. 51, no. 4, pp. 676685, 2010

[8] C. P. Panayiotopoulos, "The new ILAE report on terminology and concepts for the organization of epilepsies: critical review and contribution," Epilepsia, vol. 53, no. 3, pp. 399-404, 2012.

[9] G. Avanzini, "A sound conceptual framework for an epilepsy classification is still lacking," Epilepsia, vol. 51, no. 4, pp. 720722, 2010.

[10] R. Ottman, J. F. Annegers, N. Risch, W. A. Hauser, and M. Susser, "Relations of genetic and environmental factors in the etiology of epilepsy," Annals of Neurology, vol. 39, no. 4, pp. 442-449, 1996.

[11] X. Wang and Y. Lu, "Genetic etiology of new forms of familial epilepsy," Frontiers in Bioscience, vol. 13, no. 8, pp. 3159-3167, 2008.

[12] M. J. Martínez, M. A. López-Aríztegui, N. Puente, I. Rubio, and M. I. Tejada, "CDKL5 gene status in female patients with epilepsy and Rett-like features: two new mutations in the catalytic domain," BMC Medical Genetics, vol. 13, article 68, 2012.

[13] A. Vezzani, J. French, T. Bartfai, and T. Z. Baram, "The role of inflammation in epilepsy," Nature Reviews Neurology, vol. 7, no. 1, pp. 31-40, 2011.

[14] Y. Takahashi, K. Matsuda, Y. Kubota et al., "Vaccination and infection as causative factors in Japanese patients with Rasmussen syndrome: molecular mimicry and HLA class I," Clinical and Developmental Immunology, vol. 13, no. 2-4, pp. 381-387, 2006.

[15] W. H. Theodore, L. Epstein, W. D. Gaillard, S. Shinnar, M. S. Wainwright, and S. Jacobson, "Human herpes virus 6B: a possible role in epilepsy?” Epilepsia, vol. 49, no. 11, pp. 1828-1837, 2008.

[16] J. E. Libbey and R. S. Fujinami, "Neurotropic viral infections leading to epilepsy: focus on Theiler's murine encephalomyelitis virus," Future Virology, vol. 6, no. 11, pp. 1339-1350, 2011.

[17] Y. Bozzi, S. Casarosa, and M. Caleo, "Epilepsy as a neurodevelopmental disorder," Frontiers in Psychiatry, vol. 3, article 19, 2012.

[18] P. Sgadò, M. Dunleavy, S. Genovesi, G. Provenzano, and Y. Bozzi, "The role of GABAergic system in neurodevelopmental disorders: a focus on autism and epilepsy," International Journal of Physiology, Pathophysiology and Pharmacology, vol. 3, no. 3, pp. 223-235, 2011.

[19] R. Tuchman and M. Cuccaro, "Epilepsy and autism: neurodevelopmental perspective," Current Neurology and Neuroscience Reports, vol. 11, no. 4, pp. 428-434, 2011.
[20] R. J. Hagerman, "Epilepsy drives autism in neurodevelopmental disorders," Developmental Medicine and Child Neurology, vol. 55, no. 2, pp. 101-102, 2013.

[21] A. M. van Eeghen, M. B. Pulsifer, V. L. Merker et al., "Understanding relationships between autism, intelligence, and epilepsy: a cross-disorder approach," Developmental Medicine and Child Neurology, vol. 55, no. 2, pp. 146-153, 2013.

[22] S. N. Rakhade and F. E. Jensen, "Epileptogenesis in the immature brain: emerging mechanisms," Nature Reviews Neurology, vol. 5, no. 7, pp. 380-391, 2009.

[23] C. M. Dubé, A. L. Brewster, C. Richichi, Q. Zha, and T. Z. Baram, "Fever, febrile seizures and epilepsy," Trends in Neurosciences, vol. 30, no. 10, pp. 490-496, 2007.

[24] J. Palace and B. Lang, "Epilepsy: an autoimmune disease?" Journal of Neurology Neurosurgery and Psychiatry, vol. 69, no. 6, pp. 711-714, 2000.

[25] S. Najjar, M. Bernbaum, G. Lai, and O. Devinsky, "Immunology and epilepsy," Reviews in Neurological Diseases, vol. 5, no. 3, pp. 109-116, 2008.

[26] E. Pineda, D. Shin, S. J. You, S. Auvin, R. Sankar, and A. Mazarati, "Maternal immune activation promotes hippocampal kindling epileptogenesis in mice," Annals of Neurology, vol. 74, no. 1, pp. 11-19, 2013.

[27] R. Nabbout, "Autoimmune and inflammatory epilepsies," Epilepsia, vol. 53, no. 4, pp. 58-62, 2012.

[28] A. Vincent and P. B. Crino, "Systemic and neurologic autoimmune disorders associated with seizures or epilepsy," Epilepsia, vol. 52, supplement 3, pp. 12-17, 2011.

[29] T. Granata, H. Cross, W. Theodore, and G. Avanzini, "Immunemediated epilepsies," Epilepsia, vol. 52, supplement 3, pp. 5-11, 2011.

[30] A. Vezzani and S. Rüegg, "The pivotal role of immunity and inflammatory processes in epilepsy is increasingly recognized: introduction," Epilepsia, vol. 52, supplement 3, pp. 1-4, 2011.

[31] N. Specchio, L. Fusco, D. Claps, and F. Vigevano, "Epileptic encephalopathy in children possibly related to immunemediated pathogenesis," Brain \& Development, vol. 32, no. 1, pp. 51-56, 2010.

[32] K. M. Rodgers, M. R. Hutchinson, A. Northcutt, S. F. Maier, L. R. Watkins, and D. S. Barth, "The cortical innate immune response increases local neuronal excitability leading to seizures," Brain, vol. 132, no. 9, pp. 2478-2486, 2009.

[33] H. J. M. Majoie, M. de Baets, W. Renier, B. Lang, and A. Vincent, "Antibodies to voltage-gated potassium and calcium channels in epilepsy," Epilepsy Research, vol. 71, no. 2-3, pp. 135-141, 2006.

[34] K. McKnight, Y. Jiang, Y. Hart et al., "Serum antibodies in epilepsy and seizure-associated disorders," Neurology, vol. 65, no. 11, pp. 1730-1736, 2005.

[35] C. G. Bien and A. Vincent, "Immune-mediated pediatric epilepsies," Handbook of Clinical Neurology, vol. 111, pp. 521-531, 2013.

[36] M. Falip, M. Carreño, J. Miró et al., "Prevalence and immunological spectrum of temporal lobe epilepsy with glutamic acid decarboxylase antibodies," European Journal of Neurology, vol. 19, no. 6, pp. 827-833, 2012.

[37] A. Boronat, L. Sabater, A. Saiz, J. Dalmau, and F. Graus, "GABA ${ }_{B}$ receptor antibodies in limbic encephalitis and antiGAD-associated neurologic disorders," Neurology, vol. 76, no. 9, pp. 795-800, 2011.

[38] V. Nociti, G. Frisullo, T. Tartaglione et al., "Refractory generalized seizures and cerebellar ataxia associated with antiGAD antibodies responsive to immunosuppressive treatment," European Journal of Neurology, vol. 17, no. 1, p. e5, 2010. 
[39] C. I. Akman, M. C. Patterson, A. Rubinstein, and R. Herzog, "Limbic encephalitis associated with anti-GAD antibody and common variable immune deficiency," Developmental Medicine and Child Neurology, vol. 51, no. 7, pp. 563-567, 2009.

[40] E. Krastinova, M. Vigneron, P. Le Bras, J. Gasnault, and C. Goujard, "Treatment of limbic encephalitis with anti-gliomainactivated 1 (LGI1) antibodies," Journal of Clinical Neuroscience, vol. 19, no. 11, pp. 1580-1582, 2012.

[41] A. M. L. Quek, J. W. Britton, A. McKeon et al., "Autoimmune epilepsy: clinical characteristics and response to immunotherapy," Archives of Neurology, vol. 69, no. 5, pp. 582-593, 2012.

[42] M. Nørgaard, V. Ehrenstein, R. B. Nielsen, L. S. Bakketeig, and H. T. Sørensen, "Maternal use of antibiotics, hospitalisation for infection during pregnancy, and risk of childhood epilepsy: a population-based cohort study," PLoS ONE, vol. 7, no. 1, Article ID e30850, 2012.

[43] J. F. Bale Jr., "Fetal infections and brain development," Clinics in Perinatology, vol. 36, no. 3, pp. 639-653, 2009.

[44] C. S. Wu, L. H. Pedersen, J. E. Miller et al., "Risk of cerebral palsy and childhood epilepsy related to infections before or during pregnancy," PLoS ONE, vol. 8, no. 2, Article ID e57552, 2013.

[45] Y. Sun, M. Vestergaard, J. Christensen, A. J. Nahmias, and J. Olsen, "Prenatal exposure to maternal infections and epilepsy in childhood: a population-based cohort study," Pediatrics, vol. 121, no. 5, pp. el100-e1107, 2008.

[46] K. E. Nilsen, M. C. Walker, and H. R. Cock, "Characterization of the tetanus toxin model of refractory focal neocortical epilepsy in the rat," Epilepsia, vol. 46, no. 2, pp. 179-187, 2005.

[47] M. Mainardi, M. Pietrasanta, E. Vannini, O. Rossetto, and M. Caleo, "Tetanus neurotoxin-induced epilepsy in mouse visual cortex," Epilepsia, vol. 53, no. 7, pp. e132-e136, 2012.

[48] R. C. Wykes, J. H. Heeroma, L. Mantoan et al., "Optogenetic and potassium channel gene therapy in a rodent model of focal neocortical epilepsy," Science Translational Medicine, vol. 4, no. 161, Article ID 161ra152, 2012.

[49] W. M. Otte, P. Bielefeld, R. M. Dijkhuizen, and K. P. J. Braun, "Focal neocortical epilepsy affects hippocampal volume, shape, and structural integrity: a longitudinal MRI and immunohistochemistry study in a rat model," Epilepsia, vol. 53, no. 7, pp. 1264-1273, 2012.

[50] M. Sedigh-Sarvestani, G. I. Thuku, S. Sunderam et al., "Rapid eye movement sleep and hippocampal theta oscillations precede seizure onset in the tetanus toxin model of temporal lobe epilepsy," The Journal of Neuroscience, vol. 34, no. 4, pp. 11051114, 2014.

[51] D. Kanduc, A. Stufano, G. Lucchese, and A. Kusalik, "Massive peptide sharing between viral and human proteomes," Peptides, vol. 29, no. 10, pp. 1755-1766, 2008.

[52] G. Lucchese, A. Stufano, M. Calabro, and D. Kanduc, "Charting the peptide crossreactome between HIV-1 and the human proteome," Frontiers in Bioscience, vol. 3, no. 4, pp. 1385-1400, 2011.

[53] B. Trost, G. Lucchese, A. Stufano, M. Bickis, A. Kusalik, and D. Kanduc, "No human protein is exempt from bacterial motifs, not even one," Self/Nonself, vol. 1, no. 4, pp. 328-334, 2010.

[54] G. Lucchese, A. Stufano, and D. Kanduc, "Proposing lowsimilarity peptide vaccines against mycobacterium tuberculosis," Journal of Biomedicine and Biotechnology, vol. 2010, Article ID 832341, 8 pages, 2010.

[55] S. L. Bavaro, M. Calabrò, and D. Kanduc, "Pentapeptide sharing between Corynebacterium diphtheria toxin and the human neural protein network," Immunopharmacology and Immunotoxicology, vol. 33, no. 2, pp. 360-372, 2011.

[56] D. Kanduc, "Describing the hexapeptide identity platform between the influenza A H5N1 and Homo sapiens proteomes," Biologics, vol. 4, pp. 245-261, 2010.

[57] R. Ricco and D. Kanduc, "Hepatitis B virus and homo sapiens proteomewide analysis: a profusion of viral peptide overlaps in neuron-specific human proteins," Biologics: Targets and Therapy, vol. 4, pp. 75-81, 2010.

[58] G. Lucchese, G. Capone, and D. Kanduc, "Peptide sharing between Influenza A H1N1 hemagglutinin and human axon guidance proteins," Schizophrenia Bulletin, vol. 40, no. 2, pp. 362-375, 2014.

[59] A. Hoshino, M. Saitoh, A. Oka et al., "Epidemiology of acute encephalopathy in Japan, with emphasis on the association of viruses and syndromes," Brain \& Development, vol. 34, no. 5, pp. 337-343, 2012.

[60] D. Kanduc, "Homology, similarity, and identity in peptide epitope immunodefinition," Journal of Peptide Science, vol. 18, no. 8, pp. 487-494, 2012.

[61] D. Kanduc, "Pentapeptides as minimal functional units in cell biology and immunology," Current Protein \& Peptide Science, vol. 14, no. 2, pp. 111-120, 2013.

[62] D. B. Sant'Angelo, E. Robinson, C. A. Janeway Jr., and L. K. Denzin, "Recognition of core and flanking amino acids of MHC classII-bound peptides by the T cell receptor," European Journal of Immunology, vol. 32, no. 9, pp. 2510-2520, 2002.

[63] J. B. Rothbard and M. L. Gefter, "Interactions between immunogenic peptides and MHC proteins," Annual Review of Immunology, vol. 9, pp. 527-565, 1991.

[64] J. B. Rothbard, R. M. Pemberton, H. C. Bodmer, B. A. Askonas, and W. R. Taylor, "Identification of residues necessary for clonally specific recognition of a cytotoxic T cell determinant," The EMBO Journal, vol. 8, no. 8, pp. 2321-2328, 1989.

[65] M. J. Reddehase, J. B. Rothbard, and U. H. Koszinowski, "A pentapeptide as minimal antigenic determinant for MHC class I-restricted T lymphocytes," Nature, vol. 337, no. 6208, pp. 651653, 1989.

[66] B. Hemmer, T. Kondo, B. Gran et al., "Minimal peptide length requirements for $\mathrm{CD}^{+} \mathrm{T}$ cell clones-implications for molecular mimicry and T cell survival," International Immunology, vol. 12, no. 3, pp. 375-383, 2000.

[67] K. Landsteiner and J. van der Scheer, "On the serological specificity of peptides. III," The Journal of Experimental Medicine, vol. 69, no. 5, pp. 705-719, 1939.

[68] R. Tiwari, J. Geliebter, A. Lucchese, A. Mittelman, and D. Kanduc, "Computational peptide dissection of Melan-a/MART-1 oncoprotein antigenicity," Peptides, vol. 25, no. 11, pp. 1865-1871, 2004.

[69] S. Tanabe, "Epitope peptides and immunotherapy," Current Protein \& Peptide Science, vol. 8, no. 1, pp. 109-118, 2007.

[70] W. Zeng, J. Pagnon, and D. C. Jackson, "The C-terminal pentapeptide of LHRH is a dominant B cell epitope with antigenic and biological function," Molecular Immunology, vol. 44, no. 15, pp. 3724-3731, 2007.

[71] G. Lucchese, A. Stufano, B. Trost, A. Kusalik, and D. Kanduc, "Peptidology: short amino acid modules in cell biology and immunology," Amino Acids, vol. 33, no. 4, pp. 703-707, 2007.

[72] P. Cossette, L. Liu, K. Brisebois et al., "Mutation of GABRA1 in an autosomal dominant form of juvenile myoclonic epilepsy," Nature Genetics, vol. 31, no. 2, pp. 184-189, 2002. 
[73] K. R. Veeramah, J. E. O’Brien, M. H. Meisler et al., “De novo pathogenic SCN8A mutation identified by whole-genome sequencing of a family quartet affected by infantile epileptic encephalopathy and SUDEP," American Journal of Human Genetics, vol. 90, no. 3, pp. 502-510, 2012.

[74] T. Suzuki, A. V. Delgado-Escueta, K. Aguan et al., "Mutations in EFHC1 cause juvenile myoclonic epilepsy," Nature Genetics, vol. 36, no. 8, pp. 842-849, 2004.

[75] A. A. Mohit, J. H. Martin, and C. A. Miller, "p493F12 kinase: a novel MAP kinase expressed in a subset of neurons in the human nervous system," Neuron, vol. 14, no. 1, pp. 67-78, 1995.

[76] C. E. Stafstrom, "The role of the subiculum in epilepsy and epileptogenesis," Epilepsy Currents, vol. 5, no. 4, pp. 121-129, 2005.

[77] K. Sendrowski and W. Sobaniec, "Hippocampus, hippocampal sclerosis and epilepsy," Pharmacological Reports, vol. 65, no. 3, pp. 555-565, 2013.

[78] C. Toma, A. Hervás, B. Torrico et al., "Analysis of two languagerelated genes in Autism: a case-control association study of FOXP2 and CNTNAP2," Psychiatric Genetics, vol. 23, no. 2, pp. 82-85, 2013.

[79] T. D. Folsom and S. H. Fatemi, "The involvement of Reelin in neurodevelopmental disorders," Neuropharmacology, vol. 68, pp. 122-135, 2013.

[80] E. Romano, C. Michetti, A. Caruso, G. Laviola, and M. L. Scattoni, "Characterization of neonatal vocal and motor repertoire of reelin mutant mice," PLoS ONE, vol. 8, no. 5, Article ID e64407, 2013.

[81] S. Jeste, M. Sahin, P. Bolton, G. Ploubidis, and A. Humphrey, "Characterization of autism in young children with tuberous sclerosis complex," Journal of Child Neurology, vol. 23, no. 5, pp. 520-525, 2008.

[82] D. C. Taylor, "Schizophrenias and epilepsies: why? When? How?" Epilepsy and Behavior, vol. 4, no. 5, pp. 474-482, 2003.

[83] P. Qin, H. Xu, T. M. Laursen, M. Vestergaard, and P. B. Mortensen, "Risk for schizophrenia and schizophrenia-like psychosis among patients with epilepsy: population based cohort study," British Medical Journal, vol. 331, no. 7507, pp. 23-25, 2005.

[84] Y.-T. Chang, P.-C. Chen, I.-J. Tsai et al., "Bidirectional relation between schizophrenia and epilepsy: a population-based retrospective cohort study," Epilepsia, vol. 52, no. 11, pp. 2036-2042, 2011.

[85] M. C. Clarke, A. Tanskanen, M. O. Huttunen, M. Clancy, D. R. Cotter, and M. Cannon, "Evidence for shared susceptibility to epilepsy and psychosis: a population-based family study," Biological Psychiatry, vol. 71, no. 9, pp. 836-839, 2012.

[86] N. G. Cascella, D. J. Schretlen, and A. Sawa, "Schizophrenia and epilepsy: is there a shared susceptibility?" Neuroscience Research, vol. 63, no. 4, pp. 227-235, 2009.

[87] A. Sette, A. Vitiello, B. Reherman et al., "The relationship between class I binding affinity and immunogenicity of potential cytotoxic T cell epitopes," Journal of Immunology, vol. 153, no. 12, pp. 5586-5592, 1994.

[88] K. D. Moudgil and E. E. Sercarz, "Understanding crypticity is the key to revealing the pathogenesis of autoimmunity," Trends in Immunology, vol. 26, no. 7, pp. 355-359, 2005.

[89] P. Eggleton, R. Haigh, and P. G. Winyard, "Consequence of neoantigenicity of the 'altered self"' Rheumatology, vol. 47, no. 5, pp. 567-571, 2008.

[90] D. Kanduc, "Peptide cross-reactivity: the original sin of vaccines," Frontiers in Bioscience, vol. 4, pp. 1393-1401, 2012. 


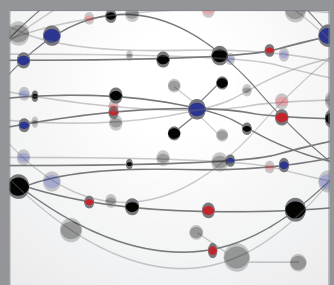

The Scientific World Journal
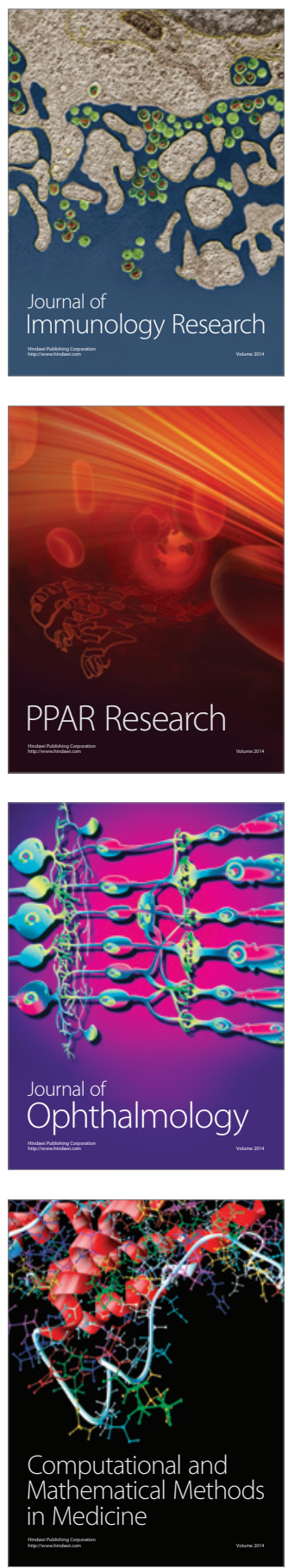

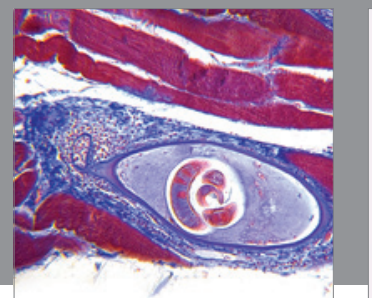

Gastroenterology

Research and Practice
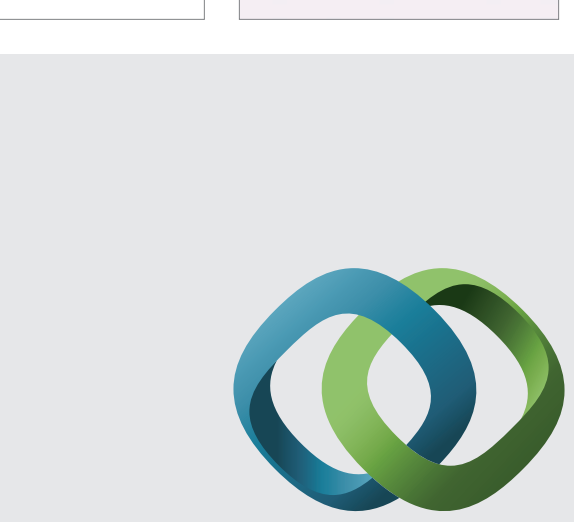

\section{Hindawi}

Submit your manuscripts at

http://www.hindawi.com
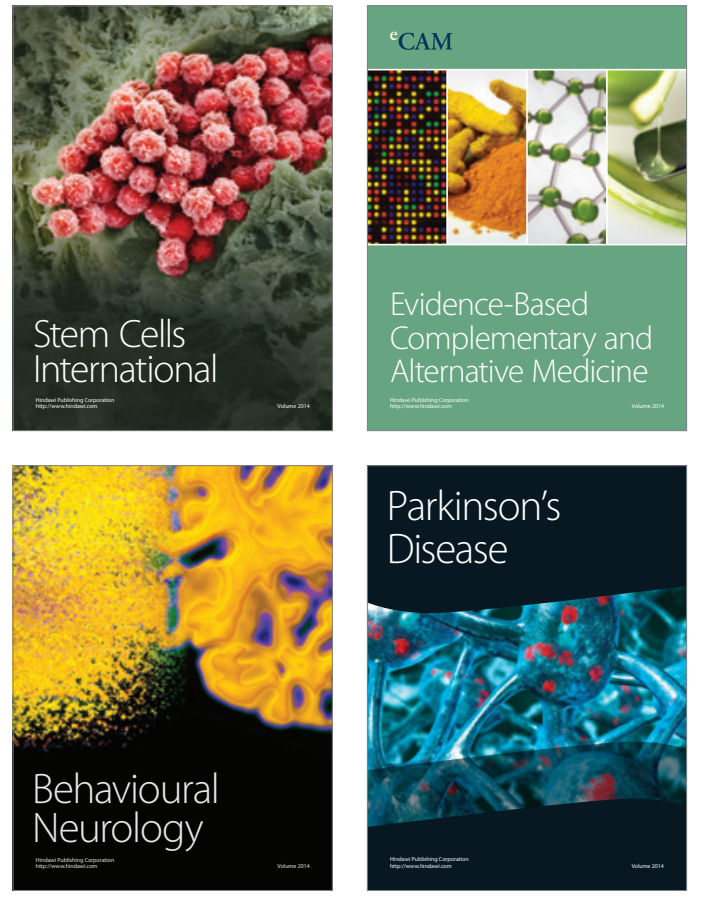
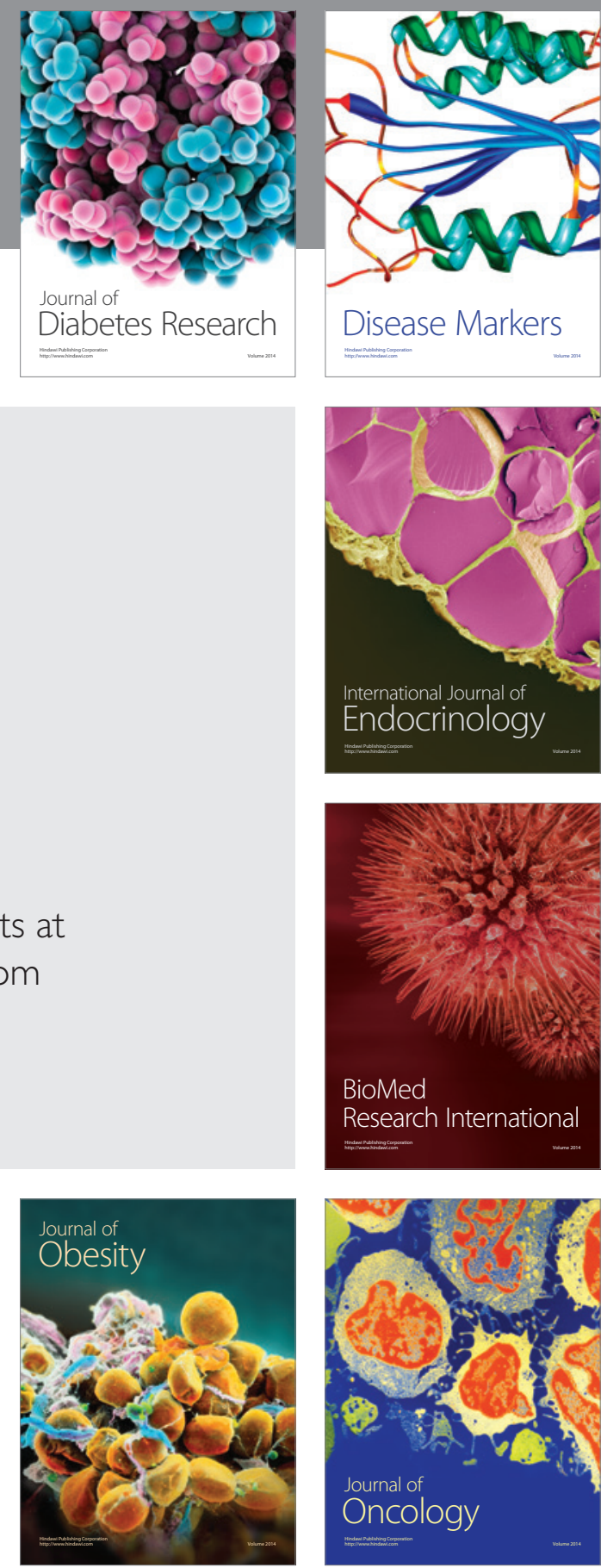

Disease Markers
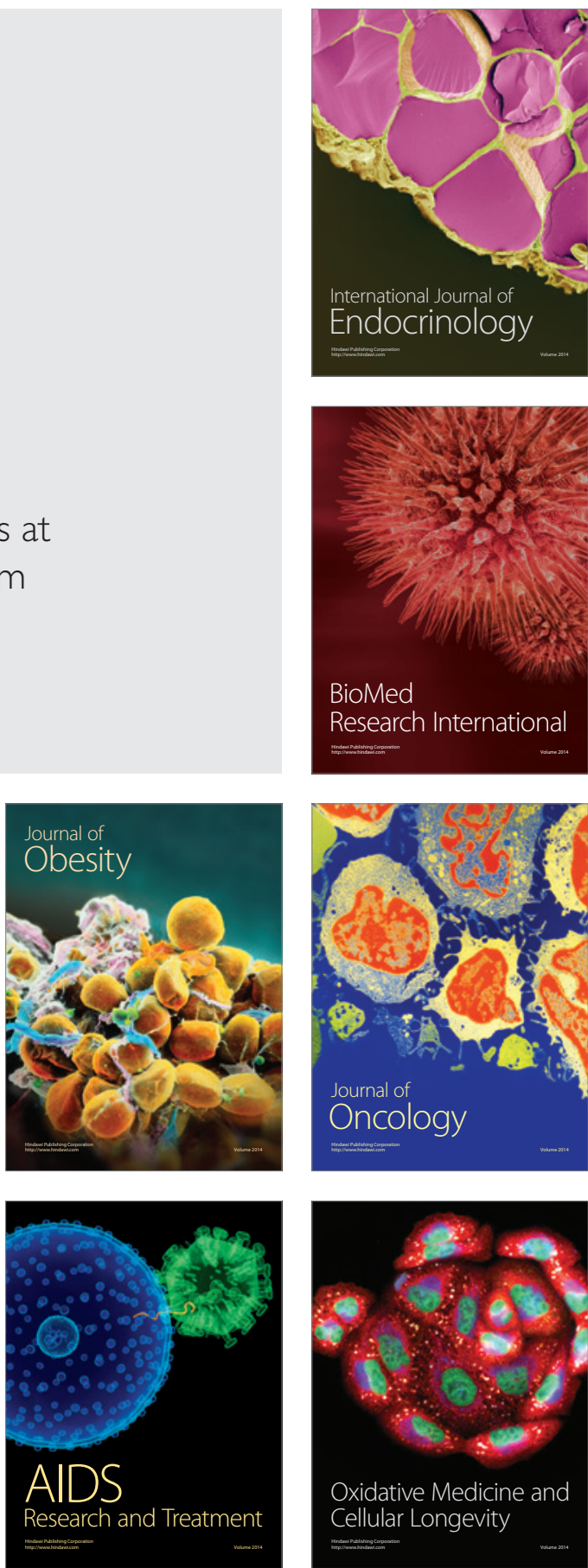